\title{
Stability results for Houghton groups
}

\author{
PETER PATZT \\ XiaOlei Wu
}

\begin{abstract}
We prove homological stability for a twisted version of the Houghton groups and their multidimensional analogues. Based on this, we can describe the homology of the Houghton groups and that of their multidimensional analogues over constant noetherian coefficients as an essentially finitely generated FI-module.
\end{abstract}

18D10, 20J06, 55U05

\section{Introduction}

The Houghton groups were first introduced in [8] by Houghton. In [3], K Brown proved that Houghton's group $\mathcal{H}_{n}$ is of type $F P_{n-1}$ but not $F P_{n}$. The group $\mathcal{H}_{n}$ can be defined as follows (see [3, Section 5]).

Let $\mathbb{N}$ be the set of positive integers, and $[n]=\{1,2, \ldots, n\}$. Let $\mathcal{H}_{n}$ be the group of all permutations (self bijections) $g$ of $\mathbb{N} \times[n]$ such that on each copy of $\mathbb{N}, g$ is eventually a translation. More precisely, we require the following condition:

- There is an $n$-tuple $\left(d_{1}, d_{2}, \ldots, d_{n}\right) \in \mathbb{Z}^{n}$ such that for each $i \in[n]$ one has $g(x, i)=\left(x+d_{i}, i\right)$ for sufficiently large $x \in \mathbb{N}$.

We also define a twisted version $\tilde{\mathcal{H}}_{n}$ of $\mathcal{H}_{n}$ as follows. An element $g \in \tilde{\mathcal{H}}_{n}$ is a permutation of $\mathbb{N} \times[n]$ such that the following condition is true:

- There is an $n$-tuple $\left(d_{1}, d_{2}, \ldots, d_{n}\right) \in \mathbb{Z}^{n}$ and $\sigma \in \mathfrak{S}_{n}$ such that for each $i \in[n]$, one has $g(x, i)=\left(x+d_{i}, \sigma(i)\right)$ for sufficiently large $x \in \mathbb{N}$.

We can embed the symmetric group $\mathfrak{S}_{n}$ into the set of permutations of $\mathbb{N} \times[n]$ by only acting on $[n]$. That is to say, an element of $\sigma \in \mathfrak{S}_{n}$ acts on $(x, i) \in \mathbb{N} \times[n]$ by $\sigma(x, i)=(x, \sigma(i))$. Then $\widetilde{\mathcal{H}}_{n}$ is generated by $\mathcal{H}_{n}$ and $\mathfrak{S}_{n}$. In fact, $\mathcal{H}_{n}$ is a normal subgroup of $\widetilde{\mathcal{H}}_{n}$ and $\widetilde{\mathcal{H}}_{n} \cong \mathcal{H}_{n} \rtimes \mathfrak{S}_{n}$. Since $\mathcal{H}_{n}$ is a finite index subgroup of $\widetilde{\mathcal{H}}_{n}$, the twisted Houghton group $\widetilde{\mathcal{H}}_{n}$ has the same finiteness properties as $\mathcal{H}_{n}$ (see Chapter VIII, Proposition 5.1 of Brown [2]).

The inclusion map $\mathbb{N} \times[n] \subset \mathbb{N} \times[n+1]$ induces a map from $\tilde{\mathcal{H}}_{n}$ to $\tilde{\mathcal{H}}_{n+1}$. We will prove that these groups satisfy homological stability. Indeed we want to prove this for 
a multidimensional version of the Houghton groups. These groups were defined by Bieri and Sach recently in [1], where they proved the multidimensional version has similar finiteness properties to the original Houghton groups.

Let us first define $\tilde{\mathcal{H}}_{k, n}$, the $k$-dimensional version of the twisted Houghton group. This shall be a subgroup of permutations on $\mathbb{N}^{k} \times[n]$. We will call a subset $X \subset \mathbb{N}^{k}$ an $r$-dimensional ray if there is a point $x \in \mathbb{N}^{k}$ and an $r$-subset $T \subset[k]$ such that

$$
X=\left\{y \in \mathbb{N}^{k} \mid y_{j} \geq x_{j} \text { for all } j \in T, y_{j}=x_{j} \text { for all } j \notin T\right\} .
$$

Let $\widetilde{\mathcal{H}}_{k, n}$ be the group of all permutations that are translations on all rays of a finite partition of $\mathbb{N}^{k} \times[n]$ into rays. By a translation we mean a map $f: X \rightarrow \mathbb{N}^{k} \times[n]$ given by $f(x, i)=\left(x+d, i+d_{0}\right)$ for $d \in \mathbb{Z}^{k}$ and $d_{0} \in \mathbb{Z}$. Note that 0 -dimensional rays are just points. In Bieri and Sach's notion, this is the $\operatorname{group} \operatorname{Pet}\left(\mathbb{N}^{k} \times[n]\right)$ where $\mathbb{N}^{k} \times[n] \subset \mathbb{Z}^{k+1}$, and the structure group $\mathbb{Z}^{k+1}$ acts on the lattice $\mathbb{Z}^{k+1}$ as translations.

Every finite partition of $\mathbb{N}^{k} \times[n]$ must contain exactly one $k$-dimensional ray for every copy of $\mathbb{N}^{k}$. Therefore there is a surjection $\widetilde{\mathcal{H}}_{k, n} \rightarrow \mathfrak{S}_{n}$. Define $\mathcal{H}_{k, n}$ as the kernel of that map. Note that this short exact sequence splits. Again from the inclusion of $\mathbb{N}^{k} \times[n] \subset \mathbb{N}^{k} \times[n+1]$ we get inclusion maps $\tilde{\mathcal{H}}_{k, n} \rightarrow \widetilde{\mathcal{H}}_{k, n+1}$ and $\mathcal{H}_{k, n} \rightarrow \mathcal{H}_{k, n+1}$. Note that $\widetilde{\mathcal{H}}_{1, n}=\widetilde{\mathcal{H}}_{n}$ and $\mathcal{H}_{1, n}=\mathcal{H}_{n}$.

We now formulate our homological stability result for the twisted Houghton groups.

Theorem A The induced map

$$
H_{i}\left(\tilde{\mathcal{H}}_{k, n} ; \mathbb{Z}\right) \rightarrow H_{i}\left(\tilde{\mathcal{H}}_{k, n+1} ; \mathbb{Z}\right)
$$

is surjective if $i \leq \frac{1}{2}(n-1)$ and injective if $i \leq \frac{1}{2}(n-2)$.

Remark Here we restrict our main result to the constant coefficient $\mathbb{Z}$ case. Nevertheless, the theorem also holds for some general coefficients by applying Theorem A from Wahl and Randal-Williams [10].

Remark In Burillo, Cleary, Martino and Röver [4, Theorem 2.2], it was shown that $\operatorname{Aut}\left(\mathcal{H}_{n}\right)$ is isomorphic to $\tilde{\mathcal{H}}_{n}$. Hence Theorem A can also be understood as homological stability of the automorphism groups $\operatorname{Aut}\left(\mathcal{H}_{n}\right)$.

We have a natural action of the symmetric groups on the homology groups of the Houghton groups. Therefore we should not expect homological stability. However, using a result of Putman and Sam [9] we can prove a representation stability result. There have been different notions of representation stability. One that seems to imply most of them in different contexts is the structure of a finitely generated FI-module. 
Theorem B Let $R$ a commutative noetherian ring. Then for every $i, k \in \mathbb{N}$ there is an $\mathrm{FI}$-module $V$, given by $V_{n}=H_{i}\left(\mathcal{H}_{k, n} ; R\right)$, which is essentially finitely generated, ie there is a finitely generated $\mathrm{FI}-$ module $W$ and a map $W \rightarrow V$ such that $W_{n} \rightarrow V_{n}$ is surjective for all large enough $n \in \mathbb{N}$.

Remark Setting $R=\mathbb{Q}$ this immediately gives uniform representation stability by a theorem of Church, Ellenberg and Farb [5, Theorem 1.13]. For dimension $k=1$ one can see this directly, using the short exact sequence $1 \rightarrow \mathfrak{S}_{\infty} \rightarrow \mathcal{H}_{n} \rightarrow \mathbb{Z}^{n-1} \rightarrow 1$ and $H_{i}\left(\mathfrak{S}_{\infty} ; \mathbb{Q}\right)=0$.

The paper is organized as follows. In Section 1 we prove Theorem A and in Section 2 we prove Theorem B. In more detail in the first section we recall definitions and results from the categorical framework for homological stability in [10]. We prove Theorem A by constructing a homogeneous category for the twisted Houghton group $\widetilde{\mathcal{H}}_{n}$ and proving its associated simplicial complex is highly connected by applying a generalization of a proposition of Hatcher and Wahl [7, Proposition 3.5]. In the second section we quickly give the necessary background for a result of Putman and Sam [9, Theorem 5.13]. We then modify this result slightly to conclude Theorem B.

Acknowledgements The first author was supported by the Berlin Mathematical School. The second author was supported by the POINT Fellowship from the Dahlem Research School. The authors also want to thank Elmar Vogt and Nathalie Wahl for helpful discussions. The proof of Corollary 1.15 was considerably shortened after Nathalie Wahl pointed out the similarities to complete join complexes.

\section{Homological stability}

We begin with a summary of the axiomatized approach to homological stability given by Randal-Williams and Wahl. The definitions and results concerning homogeneous categories are taken from [10]. The reader is encouraged to consult the cited paper for more details.

Definition 1.1 [10, Definition 1.2] Let a monoidal category $(\mathcal{C}, \oplus, 0)$ be called homogeneous if 0 is initial in $\mathcal{C}$ and if the following two properties hold :

- H1 $\operatorname{Hom}(A, B)$ is a transitive $\operatorname{Aut}(B)$-set under composition.

- $\quad$ 2 The map $\operatorname{Aut}(A) \rightarrow \operatorname{Aut}(A \oplus B)$ taking $f$ to $f \oplus \mathrm{id}_{B}$ is injective with image

$$
\operatorname{Fix}(B):=\left\{\phi \in \operatorname{Aut}(A \oplus B) \mid \phi \circ\left(l_{A} \oplus \mathrm{id}_{B}\right)=l_{A} \oplus \operatorname{id}_{B}\right\}
$$

where $l_{A}: 0 \rightarrow A$ is the unique map. 
For a homological stability result on a sequence of automorphism groups of a homogeneous category, the connectivity of a certain simplicial complex that we define next is needed.

Definition 1.2 [10, Definitions 2.2 and 2.8] Let $A, X$ be objects of a homogeneous category $(\mathcal{C}, \oplus, 0)$. For $n \geq 1$, let $S_{n}(A, X)$ denote the simplicial complex whose vertices are the maps $f: X \rightarrow A \oplus X^{\oplus n}$ and whose $p$-simplices are $(p+1)$ sets $\left\{f_{0}, \ldots, f_{p}\right\}$ such that there exists a morphism $f: X^{\oplus p+1} \rightarrow A \oplus X^{\oplus n}$ with $f \circ i_{j}=f_{j}$ for some order on the set, where

$$
i_{j}=l_{X^{\oplus j}} \oplus \mathrm{id}_{X} \oplus l_{X^{\oplus p-j}}: X=0 \oplus X \oplus 0 \rightarrow X^{\oplus p+1} .
$$

Also define the following property for a fixed pair $(A, X)$ and a slope $k \geq 2$.

- LH3 For all $n \geq 1$, the simplicial complex $S_{n}(A, X)$ is $\frac{n-2}{k}$-connected.

Definition 1.3 [10, Definition 2.5] Let $A$ and $X$ be objects of a homogeneous category $(\mathcal{C}, \oplus, 0) . \mathcal{C}$ is called locally standard at $(A, X)$ if it satisfies the following two conditions:

- LS1 The two morphisms $l_{A} \oplus \mathrm{id}_{X} \oplus l_{X}$ and $l_{A \oplus X} \oplus \mathrm{id}_{X}$ are distinct in $\operatorname{Hom}\left(X, A \oplus X^{\oplus 2}\right)$.

- LS2 For all $n \geq 1$, the map $\operatorname{Hom}\left(X, A \oplus X^{\oplus n-1}\right) \rightarrow \operatorname{Hom}\left(X, A \oplus X^{\oplus n}\right)$ taking $f$ to $f \oplus l_{X}$ is injective.

Remark 1.4 In [10, Definition 2.2] a different semisimplicial complex is used to define LH3, but our LH3 implies theirs if $\mathcal{C}$ is symmetric and locally standard at $(A, X)$. This is shown in [10, Proposition 2.9, Theorem 2.10].

We are now ready to quote the theorem we will use.

Theorem 1.5 [10, Theorem 3.1] Let $(\mathcal{C}, \oplus, 0)$ be a symmetric homogeneous category satisfying LH3, LS1, and LS2 for a pair $(A, X)$ with slope $k \geq 2$. Then the map

$$
H_{i}\left(\operatorname{Aut}\left(A \oplus X^{\oplus n}\right) ; \mathbb{Z}\right) \rightarrow H_{i}\left(\operatorname{Aut}\left(A \oplus X^{\oplus n+1}\right) ; \mathbb{Z}\right)
$$

induced by the natural inclusion map is surjective if $i \leq n / k$, and injective if $i \leq$ $(n-1) / k$.

Finally we want to quote a construction theorem that will allow us to build a category in which $\operatorname{Aut}\left(X^{\oplus n}\right)$ are the twisted Houghton groups. 
Theorem 1.6 [10, Propositions 1.6 and 1.10, Theorem 1.8] Given a symmetric monoidal groupoid $(\mathcal{G}, \oplus, 0)$ with $\operatorname{Aut}(0)=\{\operatorname{id}\}$ and the map $\operatorname{Aut}(A) \rightarrow \operatorname{Aut}(A \oplus B)$ sending $f$ to $f \oplus \mathrm{id}_{B}$ is injective for all objects $A, B$ in $\mathcal{G}$. Assume furthermore that the underlying monoid has no zero divisors and is cancellative. Then there is a symmetric homogeneous category $\mathcal{C}$, which is defined on the same elements as $\mathcal{G}$ with homomorphism sets $\operatorname{Hom}_{\mathcal{C}}(A, B \oplus A)=\operatorname{Aut}(B \oplus A) / \operatorname{Aut}(B)$ and empty if the codomain is not isomorphic to any such sum.

Let us fix a dimension $k \in \mathbb{N}$. In this section, we want to prove homological stability for the $k$-dimensional twisted Houghton groups. In order to apply Theorem 1.5, we need to introduce a symmetric homogeneous category that can be constructed using Theorem 1.6. Then it suffices to prove LH3, LS1, and LS2.

Let $\mathcal{G}_{\mathcal{H}}$ be the groupoid whose objects are the nonnegative integers such that its morphisms are all automorphisms with $\operatorname{Aut}(0)=\{\mathrm{id}\}$ and $\operatorname{Aut}(n)=\widetilde{\mathcal{H}}_{k, n}$ for $n \geq 1$. This groupoid is symmetric monoidal with the usual addition on the integers, which has no zero divisors and is cancellative. This can be seen with the map

$$
\operatorname{Aut}(m) \times \operatorname{Aut}(n) \rightarrow \operatorname{Aut}(m+n),
$$

where we want $g \in \operatorname{Aut}(m)$ to act as usual on $\mathbb{N}^{k} \times\{1, \ldots, m\}$ and $g^{\prime} \in \operatorname{Aut}(n)$ to act on $\mathbb{N}^{k} \times\{m+1, \ldots, m+n\}$. Since $g$ and $g^{\prime}$ commute, this defines a monoidal structure on $\mathcal{G}_{\mathcal{H}}$. The map

$$
\left((x, i) \mapsto\left\{\begin{array}{ll}
(x, i+n) & \text { for } i \leq m \\
(x, i-m) & \text { for } i>m
\end{array}\right) \in \operatorname{Aut}(m+n)\right.
$$

defines a symmetry.

Let $\mathcal{C}_{\mathcal{H}}$ be the homogeneous category constructed by Theorem 1.6. The next lemma will help us understand the morphism sets $\operatorname{Hom}_{\mathcal{C}_{\mathcal{H}}}(m, n)$ better.

Lemma 1.7 Let $m<n$, then $\operatorname{Hom}_{\mathcal{C}_{\mathcal{H}}}(m, n)$ can be naturally identified with the set of injections $g$ from $\mathbb{N}^{k} \times[m]$ to $\mathbb{N}^{k} \times[n]$ which are translations on every ray of a finite partition of $\mathbb{N}^{k} \times[m]$ into rays.

Proof From $m<n$, we have that $\operatorname{Hom}_{\mathcal{C}_{\mathcal{H}}}(m, n)=\widetilde{\mathcal{H}}_{k, n} / \widetilde{\mathcal{H}}_{k, n-m}$ by Theorem 1.6. We define a map $F$ from these injections to $\widetilde{\mathcal{H}}_{k, n} / \widetilde{\mathcal{H}}_{k, n-m}$. Given any injection from $\mathbb{N}^{k} \times[m]$ to $\mathbb{N}^{k} \times[n]$ that is a translation on every ray of a finite ray partition of $\mathbb{N}^{k} \times[m]$, one easily extends it to a permutation of $\mathbb{N}^{k} \times[n]$ that is a translation on every ray of a finite partition of $\mathbb{N}^{k} \times[n]$ into rays. Given two elements $g_{1}, g_{2} \in \widetilde{\mathcal{H}}_{k, n}$ extending the same injection then they coincide on the first $m$ copies of $\mathbb{N}^{k}$. Let 
$h=g_{2}^{-1} g_{1}$ which acts trivially on $\mathbb{N}^{k} \times[m]$. Thus that $h \in \tilde{\mathcal{H}}_{k, n-m}$, and so the given map $F$ is well defined.

Given an element $g \in \widetilde{\mathcal{H}}_{k, n}$, we can restrict it to $\mathbb{N}^{k} \times[m]$ to get an injection that is a translation on rays. Thus $F$ is surjective. Because every element in a coset has the same restriction the map $F$ is injective.

Let us choose $X=A=1$. From the previous lemma $\mathcal{C}_{\mathcal{H}}$ is clearly locally standard at $(A, X)$. We can apply Theorem 1.5 to get Theorem $\mathrm{A}$, if the corresponding simplicial complex $S_{n}(1,1) \cong S_{n+1}(0,1)$ (see Definition 1.2) is $\frac{n-2}{2}$-connected. Let us abbreviate $S_{n}(0,1)$ by $S_{n}$. Let us prove a few properties about simplicial complexes and come back to $S_{n}$ again later.

Definition 1.8 A simplicial complex $K$ is called weakly Cohen-Macaulay of dimension $n$ if it is $(n-1)$-connected and the link of any $p$-simplex is $(n-p-2)$-connected. In this case, we write $\operatorname{wCM}(K) \geq n$.

Remark 1.9 Note that $(-1)$-connected is defined to mean nonempty. This implies that $K$ is at least of dimension $n$. Note also that a weakly Cohen-Macaulay complex of dimension $n$ is weakly Cohen-Macaulay of dimension $m \leq n$.

Definition 1.10 Let $\pi: Y \rightarrow X$ be a surjective simplicial map between simplicial complexes. Let $S$ be a subset of the vertices of $Y$. We call a section $\rho: X \rightarrow Y$ of $\pi$ an $S$-section if for all simplices $\sigma$ in the span of $S$ and every simplex $\tau$ in $X$ we have

$$
\tau \subset \mathrm{Lk}_{X} \pi \sigma \Longleftrightarrow \rho \tau \subset \mathrm{Lk}_{Y} \sigma
$$

where $\operatorname{Lk}_{Y} \sigma$ denotes the link of $\sigma$ in $Y$. We call $\pi$ a fin-retraction if there exists an $S$-section for every finite set of vertices of $Y$.

Remark 1.11 When Hatcher and Wahl call $Y$ a complete join complex over $X$ (see [7, Definition 3.2]), there is an $S$-section where $S$ is the set of all vertices of $Y$. In particular, then $Y \rightarrow X$ is a fin-retraction. The proof of our next proposition is a generalization of [7, Proposition 3.5].

Proposition 1.12 If $\pi: Y \rightarrow X$ is a fin-retraction and $\mathrm{wCM}(X) \geq n$, then $Y$ is $(n-1)-$ connected. If $\pi$ is simplexwise injective (ie, links map to links), then $\operatorname{wCM}(Y) \geq n$.

Proof We first prove that $Y$ is $(n-1)$-connected. We need to prove that every map $f: S^{k} \rightarrow Y$ with $k \leq n-1$ homotopes to a constant map. We may assume that $f$ is simplicial. Let $S$ be the set of images of the vertices in $S^{k}$ and $\rho: X \rightarrow Y$ 
an $S$-section. Then $\rho X$ is an isomorphic image of $X$ in $Y$. If we can homotope $f$ to land in $\rho X$, we have proved $(n-1)$-connectedness.

Call a simplex of $Y$ bad if it lies in the complement of $\rho X$. Let $\sigma$ be a simplex of $S^{k}$ with maximal dimension $q$ such that $f(\sigma)$ is a bad simplex of $Y$, say of dimension $p \leq q$. By maximality, $f$ maps the link of $\sigma$ to $\rho X$. This implies that every simplex in $\operatorname{Lk}_{Y} f \sigma$ is the image of a simplex $\tau$ of $X$ under $\rho$. But $\rho \tau$ will be sent to $\pi \rho \tau=\tau$ in $X$, which is in $\operatorname{Lk}_{X} \pi f \sigma$ because $\rho$ is an $S$-section. In fact $\operatorname{Lk}_{Y} f \sigma$ is isomorphic to $\mathrm{Lk}_{X} \pi f \sigma$ because $\rho$ is an $S$-section. This link is $(n-p-2)$-connected because of our assumption on $X$ and $\operatorname{Lk}_{S^{k}} \sigma \cong S^{k-q-1}$. As $k-q-1 \leq n-p-2$, there exists a map $F: D^{k-q} \rightarrow \operatorname{Lk}_{Y} f \sigma$ extending $\left.f\right|_{\mathrm{Lk} \sigma}$. By the (relative) simplicial approximation theorem, we can extend the simplicial structure of $\mathrm{Lk}_{S^{k}} \sigma$ to $D^{k-q}$ and assume that $F$ is simplicial. Therefore there exists a simplicial map

$$
H:=\left.F * f\right|_{\sigma}: D^{k-q} * \sigma \rightarrow \overline{\operatorname{St}}(f \sigma)
$$

to the closed star of $f \sigma$. The boundary of the ball $D^{k-q} * \sigma$ is $\partial D^{k-q} * \sigma \cup D^{k-q} * \partial \sigma$. Therefore $H$ defines a homotopy from

$$
\left.f\right|_{\overline{\mathrm{St}}(\sigma)}: \overline{\mathrm{St}}(\sigma)=\partial D^{k-q} * \sigma \rightarrow \overline{\mathrm{St}}(f \sigma)
$$

to

$$
\left.F * f\right|_{\partial \sigma}: D^{k-q} * \partial \sigma \rightarrow \overline{\operatorname{St}}(f \sigma)
$$

This defines a new map $f^{\prime}$ homotopic to $f$ with fewer maximal simplices whose images are bad. Note that the simplicial structure on $S^{k}$ outside $\overline{\operatorname{St}}(\sigma)$ has not changed, however, the simplicial structure on $\overline{\operatorname{St}}(\sigma)$ has changed from $\operatorname{Lk}_{S^{k}} \sigma * \sigma$ to $D^{k-q} * \partial \sigma$. After finitely many iterations no bad simplices remain which shows that $Y$ is $(n-1)-$ connected.

Assume $\pi$ is simplexwise injective. Let $\sigma$ be a $p$-simplex in $Y$, then we need to prove that $\operatorname{Lk}_{Y} \sigma$ is $(n-p-2)$-connected. Note that the link of a $p$-simplex in $X$ is $(n-p-2)$-connected. Now we consider the restriction of $\pi$ to $\operatorname{Lk}_{Y} \sigma$ which maps to $\operatorname{Lk}_{X} \pi \sigma$ because $\pi$ is simplexwise injective. Let $\rho: X \rightarrow Y$ be an $S$-section where $S$ is the set of vertices of $\sigma$. That means for all simplices $\tau$ in $X$

$$
\tau \in \mathrm{Lk}_{X} \pi \sigma \Longleftrightarrow \rho \tau \in \mathrm{Lk}_{Y} \sigma
$$

In conclusion $\pi: \operatorname{Lk}_{Y} \sigma \rightarrow \operatorname{Lk}_{X} \pi \sigma$ is surjective. Now we want to show that the function $\pi: \operatorname{Lk}_{Y} \sigma \rightarrow \operatorname{Lk}_{X} \pi \sigma$ is a fin-retraction. Let $S$ be a finite set of vertices in $\operatorname{Lk}_{Y} \sigma$, let $\sigma^{\prime}$ be a simplex whose vertices are in $S$, and let $\tau$ be a simplex in $\operatorname{Lk}_{X} \pi \sigma$. 
Then the following equivalences hold:

$$
\begin{aligned}
\tau \subset \operatorname{Lk}_{\mathrm{Lk}_{X} \pi \sigma} \pi \sigma^{\prime} & \Longleftrightarrow \tau \subset \operatorname{Lk}_{X} \pi\left(\sigma^{\prime} * \sigma\right) \\
& \Longleftrightarrow \rho \tau \subset \operatorname{Lk}_{Y} \sigma^{\prime} * \sigma \\
& \Longleftrightarrow \rho \tau \subset \operatorname{Lk}_{\mathrm{Lk}_{Y}} \sigma^{\prime} .
\end{aligned}
$$

This proves that $\mathrm{wCM}(Y) \geq n$ if $\mathrm{wCM}(X) \geq n$.

Returning to $S_{n}$, let us define a map from $\pi: S_{n} \rightarrow \Delta^{n-1}$. By Lemma 1.7, we know every vertex of $S_{n}$ is an injection from $\mathbb{N}^{k}$ to $\mathbb{N}^{k} \times[n]$ that is a translation on rays. Since there is exactly one $k$-dimensional ray in a finite partition of $\mathbb{N}^{k}$ into rays, we can track to which copy of $\mathbb{N}^{k}$ this ray is sent. If we assume the vertex set of $\Delta^{n-1}$ is $[n]$, this gives us a map on the vertices, which uniquely extends to a simplicial map. We want to prove that $\pi^{(n-2)}: S_{n}^{(n-2)} \rightarrow\left(\Delta^{n-1}\right)^{(n-2)}$ restricted to the $(n-2)-$ skeleton is a fin-retraction. The following lemma will help us to analyze the complex $S_{n}$.

Lemma 1.13 Let $f_{1}, \ldots, f_{p+1}: \mathbb{N}^{k} \rightarrow \mathbb{N}^{k} \times[n]$ be $p+1 \leq n-1$ vertices of $S_{n}$, then they form a $p$-simplex if and only if their images in $\mathbb{N}^{k} \times[n]$ are disjoint.

Proof By the universal property of the coproduct of sets (disjoint union) we can put $f_{1}, \ldots, f_{p+1}$ together to be one map $f: \mathbb{N}^{k} \times[p+1] \rightarrow \mathbb{N}^{k} \times[n]$. Because the images of the maps are disjoint and every individual map is injective, $f$ is also injective. By Lemma 1.7, $f \in \operatorname{Hom}_{\mathcal{C}_{\mathcal{H}}}(m, n)$, which proves the lemma by Definition 1.2.

Proposition 1.14 The restriction of $\pi^{(n-2)}$ to the (n-2)-skeleton maps surjectively to the $(n-2)-$ skeleton of $\Delta^{n-1}$. Furthermore $\pi^{(n-2)}: S_{n}^{(n-2)} \rightarrow\left(\Delta^{n-1}\right)^{(n-2)}$ is a fin-retraction.

Proof $\pi^{(n-2)}$ is clearly surjective, because the identity map in $\operatorname{Hom}_{\mathcal{C}_{\mathcal{H}}}(n, n)$ defines an $(n-1)$-simplex that maps surjectively to $\Delta^{n-1}$. Thus the faces of this $(n-1)-$ simplex map surjectively onto the $(n-2)-$ skeleton of $\Delta^{n-1}$.

Let $S$ be a finite set of vertices of $S_{n}$. We want to inductively construct vertices $f_{1}, \ldots, f_{n} \in S_{n}$ such that $\rho:\left(\Delta^{n-1}\right)^{(n-2)} \rightarrow S_{n}^{(n-2)}$ mapping $i$ to $f_{i}$ gives a section of $\pi$, ie, every $n-1$ vertices form an (n-2)-simplex in $S_{n}$ and $\pi\left(f_{i}\right)=i$. We will then also prove that $\rho$ is an $S$-section.

Assume we have all $f_{i}$ with $i<p$ already constructed. To construct $f_{p}$, we consider $\mathbb{N}^{k}$ as a $k$-dimensional ray and send it to $\mathbb{N}^{k} \times\{p\}$ translating it far enough out (choosing $d \in \mathbb{Z}^{k}$ large enough) that its image is disjoint from all images of 
the vertices in $\left\{f_{i} \mid i<p\right\} \cup S-\pi^{-1}(p)$. It immediately follows that the images $f_{1}\left(\mathbb{N}^{k}\right), \ldots, f_{n}\left(\mathbb{N}^{k}\right)$ are pairwise disjoint. Thus $\rho$ gives a section.

Let $\sigma$ be a simplex in the span of $S$ and $\tau$ a simplex in the $(n-2)-$ skeleton of $\Delta^{n-1}$. The simplex $\tau$ lies in the link of $\pi \sigma$ if and only if the set of vertices of $\pi \sigma$ and $\tau$ are disjoint and the union has at most cardinality $n-1$. If this is the case, the vertices of $\sigma$ and $\rho \tau$, which are some $f_{i}$, form a simplex in $S_{n}^{(n-2)}$ by the previous lemma. Conversely, if the vertices of $\sigma$ and $\rho \tau$ form a simplex in $S_{n}^{(n-2)}$ it can at most have $n-1$ vertices, and the $k$-dimensional ray of each vertex must be sent to a different copy of $\mathbb{N}^{k}$. That means that $\pi$ sends the vertices to distinct vertices.

Corollary 1.15 The simplicial complex $S_{n}$ is $\frac{n-3}{2}$-connected.

Proof From Proposition 1.12 it follows that $S_{n}^{(n-2)}$ is $(n-3)$-connected. For $n \geq 2$, $n-3 \geq\left\lfloor\frac{n-3}{2}\right\rfloor$ implies it is in particular $\frac{n-3}{2}$-connected. $S_{1}$ is nonempty, which is needed for $n=1$.

The corollary proves that $S_{n+1}$ is $\frac{n-2}{2}$-connected, and hence $\mathbf{L H 3}$ for $S_{n}(1,1)$. Now by Theorem 1.5, this finishes the proof of Theorem A.

\section{Representation stability}

In this last section, we want to analyze the homology of the Houghton groups $\mathcal{H}_{k, n}$ and prove Theorem B that with constant noetherian coefficients their homology can be described as "essentially" finitely generated FI-modules. We will shortly explain how we consider the homology of the Houghton groups as FI-modules. Then describe the central stability theory of Putman and Sam, which they introduced in [9]. Making slight adjustments, we can use their Theorem 5.13 to prove that the homology groups of the Houghton groups are essentially finitely generated $\mathrm{FI}$-modules.

Let $\mathrm{FI}$ be the category of finite sets and injections. In fact this is the homogeneous category we get from the construction in Theorem 1.6 starting with the symmetric monoidal groupoid of finite sets and bijections. (See also [10, Section 5.1].) Let us fix a commutative noetherian ring $R$, then we call a functor from $\mathrm{FI}$ to the category of $R$-modules an $\mathrm{FI}$-module. Note that $\mathrm{FI}$ is equivalent to the full subcategory only defined on the objects $[n]$ for $n \geq 0 .{ }^{1}$ Likewise the category of FI-modules is equivalent to the functor category from the mentioned subcategory to the category of $R$-modules. By abuse of notation we will from now on refer to this subcategory when we write FI.

\footnotetext{
${ }^{1}$ Here $[0]:=\varnothing$.
} 
Let us define a functor $G$ from $\mathrm{FI}$ to the category of groups by assigning $n$ to the Houghton groups $G(n)=\mathcal{H}_{k, n}$. Given an injection $f:[m] \hookrightarrow[n]$, we define $f_{*}: \mathcal{H}_{k, m} \rightarrow \mathcal{H}_{k, n}$ sending $g \in \mathcal{H}_{k, m}$ to the element in $\mathcal{H}_{k, n}$ that maps $(x, f(i))$ to $(y, f(j))$ if $g(x, i)=(y, j)$ and leaves all other elements fixed. One easily checks that this assignment is functorial. Postcomposing with the group homology functor $H_{i}(-; R)$ will thus give an $\mathrm{Fl}-$ module, where $n$ is sent to $H_{i}\left(\mathcal{H}_{k, n} ; R\right)$.

In [9], Putman and Sam work with complemented categories, which can be shown to be homogeneous categories. A complemented category is a symmetric monoidal category $(\mathcal{C}, \oplus, 0)$ with the following properties:

(1) Every morphism in $\mathcal{C}$ is a monomorphism.

(2) 0 is initial.

(3) The map

$$
\begin{aligned}
\operatorname{Hom}(A \oplus B, C) & \rightarrow \operatorname{Hom}(A, C) \times \operatorname{Hom}(B, C), \\
f & \mapsto\left(f \circ\left(\operatorname{id}_{A} \oplus \iota_{B}\right), f \circ\left(\imath_{A} \oplus \operatorname{id}_{B}\right)\right)
\end{aligned}
$$

is injective.

(4) Every subobject has a unique complement.

A subobject of an object $X$ is an equivalence class of monomorphisms to $X$. Monomorphisms $f: A \rightarrow X$ and $f^{\prime}: A^{\prime} \rightarrow X$ are equivalent if there is an isomorphism $\psi: A \rightarrow A^{\prime}$ such that $f=f^{\prime} \circ \psi$. A complement of a subobject of an object $X$ is a subobject $g: B \rightarrow X$ if there is an isomorphism $\phi: A \oplus B \rightarrow X$ such that $f=\phi \circ\left(\mathrm{id}_{A} \oplus l_{B}\right)$ and $g=\phi \circ\left(l_{A} \oplus \mathrm{id}_{B}\right)$.

Putman and Sam say a monoidal category has a generator $X$ if all objects are isomorphic to $X^{\oplus n}$ for a unique $n \in \mathbb{N}$. We can then speak of the $X$-rank of an object. Let $\mathcal{B}$ and $\mathcal{C}$ be complemented categories with generators $X$ and $Y$, respectively. Putman and Sam call a strong monoidal functor $\Psi: \mathcal{B} \rightarrow \mathcal{C}$ a highly surjective functor if $\Psi(X)=Y$ and $\Psi_{*}: \operatorname{Aut}_{\mathcal{B}}(B) \rightarrow \operatorname{Aut}_{\mathcal{C}}(\Psi(B))$ is surjective for all objects $B \in \mathcal{B}$. In [9, Section 5] it is proven that then there is a $\mathcal{C}$-module $\mathcal{H}_{i}(\Psi ; R)$ with

$$
\mathcal{H}_{i}(\Psi ; R)_{Y^{n}}=H_{i}\left(\operatorname{ker}\left(\operatorname{Aut}_{\mathcal{B}}\left(X^{n}\right) \rightarrow \operatorname{Aut}_{\mathcal{C}}\left(Y^{n}\right)\right) ; R\right) .
$$

The homogeneous categories $\mathrm{FI}$ and $\mathcal{C}_{\mathcal{H}}$ which we defined in Section 1 are complemented categories. For $\mathrm{FI}$ this is stated in [9, Example 1.10]. For $\mathcal{C}_{\mathcal{H}}$ it is not much harder to see. It is clear that (a) holds because all morphisms are injective maps. $\mathcal{C}_{\mathcal{H}}$ being a homogeneous category implies (b). A morphism in $\operatorname{Hom}_{\mathcal{C}_{\mathcal{H}}}(a \oplus b, n)$ is a map from $\mathbb{N}^{k} \times[a] \sqcup \mathbb{N}^{k} \times[b]$ to $\mathbb{N}^{k} \times[n]$, and (c) follows from $\sqcup$ being the coproduct of sets. Finally for (d) we first observe that two maps $f: \mathbb{N}^{k} \times[m] \rightarrow \mathbb{N}^{k} \times[n]$ 
and $f^{\prime}: \mathbb{N}^{k} \times\left[m^{\prime}\right] \rightarrow \mathbb{N}^{k} \times[n]$ represent the same subobject of $n$ if and only if their image in $\mathbb{N}^{k} \times[n]$ is the same. Clearly the image is an invariant of a subobject. On the other hand if two such maps have the same image, $m=m^{\prime}$ is the number of $k$-dimensional rays that fit into the image. If $f$ and $f^{\prime}$ are translations on every ray of finite ray partitions of $\mathbb{N}^{k} \times[m]$ then this gives two finite ray partitions of the image. The intersection yields a common refinement which is again a finite ray partition of the image. Thereby we find two finite ray partitions of $\mathbb{N}^{k} \times[m]$ that by $f$ and $f^{\prime}$, respectively, map to this refinement by translations on the rays. Thus $f^{-1} \circ f^{\prime} \in \widetilde{\mathcal{H}}_{k, m}$ and both maps represent the same subobject. Then the complement of a subobject $f: \mathbb{N}^{k} \times[m] \rightarrow \mathbb{N}^{k} \times[n]$ is uniquely given by $\mathbb{N}^{k} \times[n]-\operatorname{im} f$ which can be partitioned into finitely many rays.

Similar to the discussion in the introduction we can find a functor $\Psi: \mathcal{C}_{\mathcal{H}} \rightarrow \mathrm{FI}$ sending a morphism $f: \mathbb{N}^{k} \times[m] \rightarrow \mathbb{N}^{k} \times[n]$ to the injection given by the map where the $m$-many $k$-dimensional rays are sent. This functor is in fact highly surjective, since the generator 1 is mapped to the generator 1 and $\widetilde{\mathcal{H}}_{k, n} \rightarrow \mathfrak{S}_{n}$ is surjective.

Furthermore Putman and Sam's $\mathcal{H}_{i}(\Psi ; R)$ for $\Psi: \mathcal{C}_{\mathcal{H}} \rightarrow \mathrm{FI}$ coincides with the FImodule we have defined above.

Let us introduce a notation to truncate modules $V$ over a complemented category $\mathcal{C}$ with generator $Y$. By trunc $\geq k V$, we mean the functor that sends all objects with $Y$-rank $n<k$ to zero and all other objects $A$ to $V_{A}$ as before. We call $V$ essentially finitely generated if there is some $k \in \mathbb{N}$ such that trunc $\geq k V$ is finitely generated. In [9, Section 3] Putman and Sam introduce a complex of $\mathcal{C}$-modules

$$
\Sigma_{*}: \cdots \rightarrow \Sigma_{2} V \rightarrow \Sigma_{1} V \rightarrow V,
$$

where $\left(\Sigma_{p} V\right)_{Y^{n}}$ is given by $\operatorname{Ind}_{\operatorname{Aut}\left(Y^{n-p}\right)}^{\operatorname{Aut}\left(Y^{n}\right)} V_{Y^{n-p}}$ and hence only depends on $V_{Y^{n-p}}$. Hence

$$
\left(\Sigma_{p} \operatorname{trunc}_{\geq k} V\right)_{C}=\left(\Sigma_{p} V\right)_{C}
$$

for all objects $C$ with $Y$-rank $n \geq p+k$. In the light of this observation, we can generalize their Lemma 3.6 and Theorem 3.7 to the following.

Lemma 2.1 Let $\mathcal{C}$ be a complemented category with generator $Y$ and let $V$ be a $\mathcal{C}$-module over a ring $R$. Assume that all $V_{C}$ with $C$ having large enough $Y$-rank are finitely generated $R$-modules. Then $V$ is essentially finitely generated if and only if $d:\left(\Sigma_{1} V\right)_{C} \rightarrow V_{C}$ is surjective for all $C$ with large enough $Y$-rank.

Theorem 2.2 Let $\mathcal{C}$ be a complemented category with generator $Y$. Assume that the category of $\mathcal{C}$-modules is noetherian, and let $V$ be an essentially finitely generated 
$\mathcal{C}$-module. Fix some $q \geq 1$. For all $C$ with large enough $Y$-rank, the chain complex

$$
\left(\Sigma_{q} V\right)_{C} \rightarrow\left(\Sigma_{q-1} V\right)_{C} \rightarrow \cdots \rightarrow\left(\Sigma_{1} V\right)_{C} \rightarrow V_{C} \rightarrow 0
$$

is exact.

We need one more piece of notation. Putman and Sam define a semisimplicial set $\mathfrak{I}_{C}$ for a complemented category $\mathcal{C}$ with generator $Y$ by

$$
\Im_{C, p}=\operatorname{Hom}_{\mathcal{C}}\left(Y^{p+1}, C\right) .
$$

For $C=Y^{n}$ this is the same semisimplicial set $W_{n}(0, Y)$ defined by Randal-Williams and Wahl in [10, Definition 2.1].

Let us formulate a slight variation of Theorem 5.13 from [9] that we will use to prove that for every $i \geq 0$ the $\mathrm{FI}$-module given by $H_{i}\left(\mathcal{H}_{k, n} ; R\right)$ is essentially finitely generated for every noetherian ring $R$.

Theorem 2.3 Let $\mathcal{B}$ and $\mathcal{C}$ be complemented categories with generators $X$ and $Y$, respectively. Let $\Psi: \mathcal{B} \rightarrow \mathcal{C}$ be a highly surjective functor. Fix a noetherian ring $R$ and assume the following conditions:

(1) The category of $\mathcal{C}$-modules is noetherian.

(2) For all $i \geq 0$ the $R$-module $\mathcal{H}_{i}(\Psi ; R)_{C}$ is a finitely generated $R$-module for all $C$ with large enough $Y$-rank.

(3) Fix $q \geq 0$. Then $\mathfrak{I}_{B}$ is $q$-acyclic for all objects $B \in \mathcal{B}$ with large enough $X-$ rank.

Then $\mathcal{H}_{i}(\Psi ; R)$ is an essentially finitely generated $\mathcal{C}$-module for all $i \geq 0$.

In Putman and Sam's proof of their Theorem 5.13 one can now replace their Lemma 3.6 and Theorem 3.7 by our Lemma 2.1 and Theorem 2.2, and the word finitely generated by essentially finitely generated to get a proof of Theorem 2.3 .

Theorem $\mathrm{B}$ is an application of Theorem 2.3 if we set $\mathcal{B}=\mathcal{C}_{\mathcal{H}}$, set $\mathcal{C}=\mathrm{FI}$, and set $\Psi: \mathcal{C}_{\mathcal{H}} \rightarrow \mathrm{FI}$ as above. Let us check the conditions. The first condition was proved by Church, Ellenberg, Farb, and Nagpal in [6, Theorem A]. The second condition can be directly derived from $\mathcal{H}_{k, n}$ being $F P_{n-1}$, ie, the trivial $\mathbb{Z} \mathcal{H}_{k, n}$-module $\mathbb{Z}$ admits a projective resolution which is finitely generated in dimensions $\leq n-1$. This property is proved in [3, 5.1] for the original Houghton groups and in [1, Theorem B] for $k \geq 2$. The last condition is Corollary 1.15 together with Remark 1.4 . 


\section{References}

[1] R Bieri, H Sach, Groups of piecewise isometric permutations of lattice points, preprint (2016) arXiv:1606.07728

[2] KS Brown, Cohomology of groups, Graduate Texts in Mathematics 87, Springer, New York (1982) MR672956

[3] KS Brown, Finiteness properties of groups, from: "Proceedings of the Northwestern conference on cohomology of groups", J. Pure Appl. Algebra 44 (1987) 45-75 MR885095

[4] J Burillo, S Cleary, A Martino, C E Röver, Commensurations and metric properties of Houghton's groups, preprint (2014) arXiv:1403.0026v1

[5] T Church, JS Ellenberg, B Farb, FI-modules and stability for representations of symmetric groups, Duke Math. J. 164 (2015) 1833-1910 MR3357185

[6] T Church, J S Ellenberg, B Farb, R Nagpal, FI-modules over Noetherian rings, Geom. Topol. 18 (2014) 2951-2984 MR3285226

[7] A Hatcher, N Wahl, Stabilization for mapping class groups of 3-manifolds, Duke Math. J. 155 (2010) 205-269 MR2736166

[8] C H Houghton, The first cohomology of a group with permutation module coefficients, Arch. Math. (Basel) 31 (1978/79) 254-258 MR521478

[9] A Putman, S V Sam, Representation stability and finite linear groups, preprint (2014) arXiv: 1408.3694

[10] N Wahl, O Randal-Williams, Homological stability for automorphism groups, preprint (2015) arXiv:1409.3541v2

Institut für Mathematik, Freie Universität

14195 Berlin, Germany

Max Planck Institute for Mathematics

53111 Bonn, Germany

peter.patzt@fu-berlin.de, hsiaolei.wu@mpim-bonn.mpg.de

Received: 2015-09-25 Revised: 2016-01-29 
